\title{
High-Dose Fluorescein Reveals Unusual Confocal Endomicroscope Imaging of Low-Grade Glioma
}

\author{
Evgenii Belykh ${ }^{1}$, Naomi R. Onaka ${ }^{1}$, Xiaochun Zhao ${ }^{1}$, Irakliy Abramov', \\ Jennifer M. Eschbacher ${ }^{2}$, Peter Nakaji ${ }^{2 \dagger}$ and Mark C. Preul ${ }^{1 *}$ \\ ${ }^{1}$ Department of Neurosurgery, The Loyal and Edith Davis Neurosurgical Research Laboratory, St. Joseph's Hospital and \\ Medical Center, Barrow Neurological Institute, Phoenix, AZ, United States, ${ }^{2}$ Department of Neuropathology, Barrow \\ Neurological Institute, St. Joseph's Hospital and Medical Center, Phoenix, AZ, United States
}

\section{OPEN ACCESS}

Edited by:

Talat Kiris,

Koç University, Turkey

Reviewed by:

Dan Qi,

Baylor Scott and White Health

United States

Francesco Acerbi,

Fondazione IRCCS Istituto Neurologio

Carlo Besta, Italy

*Correspondence:

Mark C. Preu

Neuropub@barrowneuro.org

†Present address:

Peter Nakaji,

Department of Neurosurgery,

University of Arizona College of

Medicine, Phoenix, AZ, United States

Specialty section

This article was submitted to Neuro-Oncology and Neurosurgical

Oncology,

a section of the journal

Frontiers in Neurology

Received: 16 February 2021 Accepted: 17 June 2021

Published: 16 July 2021

Citation:

Belykh E, Onaka NR, Zhao X, Abramov I, Eschbacher JM, Nakaji P and Preul MC (2021) High-Dose Fluorescein Reveals Unusual Confocal

Endomicroscope Imaging of Low-Grade Glioma.

Front. Neurol. 12:668656. doi: 10.3389/fneur.2021.668656
Background: Fluorescence-guided brain tumor surgery using fluorescein sodium (FNa) for contrast is effective in high-grade gliomas. However, the effectiveness of this technique for visualizing noncontrast-enhancing and low-grade gliomas is unknown. This report is the first documented case of the concurrent use of wide-field fluorescence-guided surgery and confocal laser endomicroscopy (CLE) with high-dose FNa (40 mg/kg) for intraoperative visualization of tumor tissue cellularity in a nonenhancing glioma.

Case Description: A patient underwent fluorescence-guided surgery for a left frontal lobe mass without contrast enhancement on magnetic resonance imaging. The patient received $40 \mathrm{mg} / \mathrm{kg} \mathrm{FNa}$ intravenously at the induction of anesthesia. Surgery was performed under visualization with a Yellow 560 filter and white-light wide-field imaging. Intraoperative CLE produced high-quality images of the lesion $1.5 \mathrm{~h}$ after FNa injection. Frozen-section analysis demonstrated findings comparable to those of intraoperative CLE visualization and consistent with World Health Organization (WHO) glioma grades II-III. The patient recovered without complications. Analysis of the permanent histologic sections identified the tumor as an anaplastic oligodendroglioma, IDH-mutant, 1p/19q co-deleted, consistent with WHO grade III because of discrete foci of hypercellularity and increased mitotic figures, but large regions of the lesion were low grade.

Conclusions: The use of high-dose FNa in this patient with a nonenhancing borderline low-grade/high-grade glioma produced actionable wide-field fluorescence imaging using the operating microscope and improved CLE visualization of tumor cellularity. Higher doses of FNa for intraoperative CLE imaging and possible simultaneous wide-field fluorescence surgical guidance in nonenhancing gliomas merit further investigation.

Keywords: confocal laser endomicroscopy, fluorescein sodium, fluorescence-guided surgery, low-grade glioma, nonenhancing glioma, oligodendroglioma

\section{INTRODUCTION}

Fluorescein sodium (FNa) is a fluorescent biomarker available as a water-soluble dye that has been widely used in ophthalmology since the early 1960s. Moore et al. (1) reported anecdotal clinical use of fluorescein to help localize intracranial neoplasms as early as 1948. Although FNa has demonstrated utility in increasing the extent of resection in high-grade gliomas (HGG), its 
efficacy has not been well-elucidated in low-grade gliomas (LGG), and there is currently a paucity of investigation on what FNa-guided resection may reveal when used for LGG (2-5). In a systematic review of fluorescence-guided glioma surgery, Senders et al. (6) identified 11 studies describing the use of FNa in glioma resection. All 11 studies included patients with HGG, whereas only three studies included patients with LGG.

$\mathrm{FNa}$ is commonly used for retinal angiographic procedures, and it has been shown to have few side effects with oral ingestion; the most common adverse effects are nausea and vomiting $(7,8)$. Additional adverse effects of intravenous FNa include postoperative yellow discoloration of urine at lower doses, such as $3-4 \mathrm{mg} / \mathrm{kg}$, and skin or sclera discoloration at higher doses; nonetheless, overall, it is confirmed to be safe for patients (9). Only two cases of anaphylactic reactions have been reported after neurosurgical administration of $\mathrm{FNa}$, both with an FNa dose of $20 \mathrm{mg} / \mathrm{kg}(10,11)$.

FNa is useful in identifying intracranial malignancies because it extravasates from cerebral vessels in places where the bloodbrain barrier has been damaged. This mechanism allows the dye to concentrate, localizing the area where tumor invasion has disrupted vascular integrity. The pattern of FNa extravasation is relatively unique in $\mathrm{HGG}$ compared to that in simple surgical trauma to brain tissue, and the pattern indicates a disruption in the blood-brain barrier (12). FNa is excited by light in 460-500 $\mathrm{nm}$ wavelengths, and it emits radiation in the $540-690 \mathrm{~nm}$ range. Light filters, such as the Yellow 560 filter (Carl Zeiss Meditec AG, Oberkochen, Germany), on surgical microscopes help to visualize the fluorescing tissue. However, visualization and surgical maneuvering during resection often require switching between the filter and wide-field white-light illumination. The range of $\mathrm{FNa}$ doses used for neurosurgical application has varied and has been subjectively divided into a "low-dose" (1-10 $\mathrm{mg} / \mathrm{kg})$ range that requires a fluorescence detection module on a neurosurgical operating microscope for identification and a "high-dose" (15-20 mg/kg or greater) range in which the naked eye can detect the fluorescent staining even without a fluorescence detection module (13).

In conjunction with dedicated fluorescein filters on surgical microscopes, the increasing use of intraoperative histopathologic examination with confocal laser endomicroscopy (CLE) using $\mathrm{FNa}$ has produced a tool for optically interrogating gliomas with a sensitivity and specificity comparable to the examination of a frozen section (14-18). Our institutional experience with CLE and low-dose $\mathrm{FNa}$ has been similar to that of others, albeit inconsistent for nonenhancing lesions and LGG. However, in this case report, we describe a situation in which high-dose $\mathrm{FNa}$ was administered during resection of an oligodendroglioma with the use of CLE, which produced unusually clear images of the LGG. This report contributes to the limited evidence for the possible use of $\mathrm{FNa}$ to visualize

\footnotetext{
Abbreviations: CLE, confocal laser endomicroscopy; FET-PET, ${ }^{18}$ F-fluoroethylL-tyrosine-positron emission tomography; FLAIR, fluid-attenuated inversion recovery; FNa, fluorescein sodium; HGG, high-grade gliomas; IDH, isocitrate dehydrogenase; LGG, low-grade gliomas; MRI, magnetic resonance imaging; WHO, World Health Organization; 5-ALA, 5-aminolevulinic acid.
}

LGG, particularly with the aid of CLE. The CLE images indicate that a relatively high dose of $\mathrm{FNa}$ (e.g., $20-40 \mathrm{mg} / \mathrm{kg}$ ) may produce a significant benefit in identifying tumors heretofore not labeled well with lower doses of FNa during fluorescenceguided surgery.

\section{CASE DESCRIPTION}

A patient with a 4-year history of headaches was admitted for new-onset generalized seizure. Magnetic resonance imaging (MRI) showed a nonenhancing $1.6 \times 1.3-\mathrm{cm}$ heterogeneous posterior left frontal lobe mass just anterior to the precentral gyrus, with a surrounding abnormal high signal on T2-weighted and fluid-attenuated inversion recovery (FLAIR) imaging, interpreted as probably representing edema (Figure 1). The most likely differential diagnoses considered were metastatic disease and primary brain lesion. The patient gave voluntary informed consent to participate in the CLE imaging study, which was approved by the St. Joseph's Hospital and Medical Center Institutional Review Board (No. 10BN130). In the operating room, FNa was administered to the patient shortly after the induction of anesthesia. However, instead of the typical dose of 2-5 mg/kg, a higher dose of $40 \mathrm{mg} / \mathrm{kg}$ was administered. This higher dose was within the acceptable dosing range for FNa. When the tumor area was mapped for motor function, both the posterior and anterior regions immediately adjacent to the tumor showed some motor function. Yellow discoloration of the tissue was observed because of the higher $\mathrm{FNa}$ dose (Figures 2A,B). The dye concentrated in the area of the tumor, which allowed visualization of its borders, and the tumor was resected completely.

Four biopsies from within the tumor region (the last obtained about $1.5 \mathrm{~h}$ after FNa administration) were subjected to immediate intraoperative ex vivo CLE imaging (Convivo, Carl Zeiss Meditec AG). CLE imaging demonstrated hypercellular brain architecture, with abnormal cells suggestive of a cellular tumor in all biopsy specimens (Figures 2C-G). Histologic findings on frozen sections were consistent with glioma grades II-III, with the wide area majority characteristic of grade II glioma (Figure 3). Further histologic assessment of the permanent sections revealed variable cellular and infiltrative glioma with rounded oligodendroglial morphology and prominent perinuclear halos. The tumor was assigned the overall classification of anaplastic oligodendroglioma, isocitrate dehydrogenase (IDH)-mutant, $1 \mathrm{p} / 19 \mathrm{q}$ codeleted, with ATRX expression because of the regions of more aggressive heterogeneity (discrete foci of hypercellularity and increased mitotic figures) but also extensive areas of lower-grade tumor.

CLE concurrent with high-dose FNa provided extremely clear images of cellular architecture, mitotic figures, endothelium of vessels, and swollen axons (Figure 4). The brightness and clarity of the CLE images revealed a distinct morphologic appearance not typically observed with lower-dose FNa, especially $1.5 \mathrm{~h}$ or longer after administration (but often in even less time). The patient tolerated this dose well, and the patient's postoperative yellowish skin discoloration resolved rapidly. 

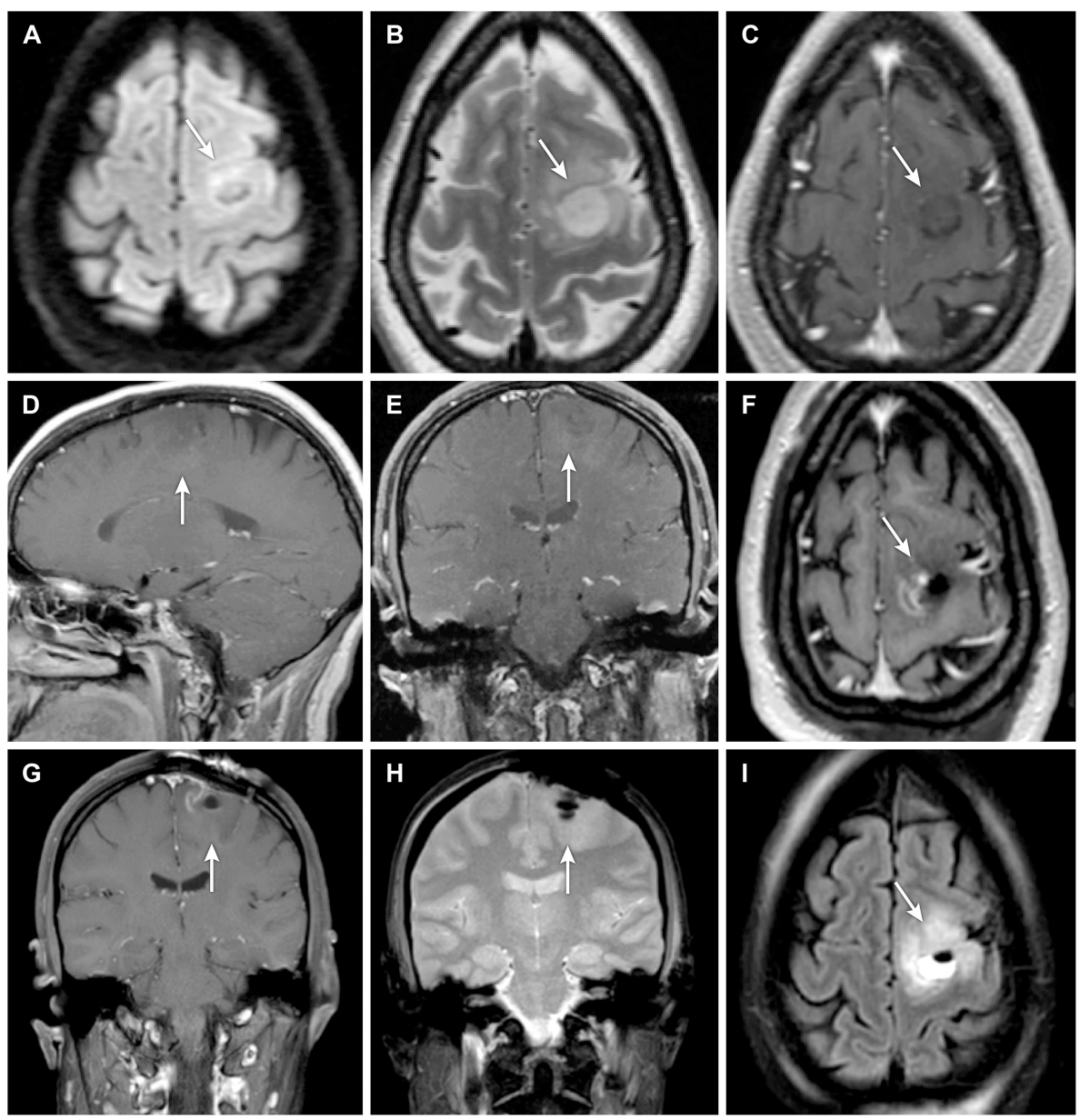

FIGURE 1 | (A-E) Preoperative and (F-I) postoperative magnetic resonance imaging (MRI) of a patient with a nonenhancing lesion (A-I, arrows) in the left precentral gyrus. Preoperative MRIs shown are (A) axial diffusion-weighted, (B) axial T2-weighted, (C) axial T1-weighted, (D) sagittal T1-weighted with contrast, and (E) coronal T1-weighted with contrast. Postoperative MRls shown are (F) axial T1-weighted with contrast, (G) coronal T1-weighted with contrast, (H) coronal diffusion-weighted, and (I) axial diffusion-weighted. Used with permission from Barrow Neurological Institute, Phoenix, Arizona.

\section{DISCUSSION}

Gliomas are classified according to WHO grades I-IV, with LGG encompassing grades I and II. Still, they are representative of a heterogeneous group of tumors with wide variability in terms of prognosis and treatment. In the case description, we present the immunohistochemistry and pathologic characteristics identifying the tumor as an anaplastic oligodendroglioma, IDH-mutant, $1 \mathrm{p} / 19 \mathrm{q}$ codeleted, with ATRX expression isolated within a vast majority of areas that were lower-grade glioma. Although the tumor was classified as a grade III glioma because of regions of increased aggressive characteristics, the prognostic significance of grade II vs. III in this tumor class compared with that of a nonanaplastic oligodendroglioma with an otherwise similar profile is not clearly understood, with the diagnostic criteria for histopathologic grading introducing subjectivity in interobserver variability in grading these lesions (19).

The tumor in this patient was categorized as grade II rather than grade III in the initial frozen-section analysis. It was noncontrast enhancing, which is a characteristic most often observed with LGG. The vast majority of this tumor was of a low-grade II glioma. The tumor was assigned the grade III classification because some localized regions were interpreted to be more aggressive. Low-grade tumors are often show localized areas or have heterogeneous regions in transition to more aggressive states.

Despite the grade III classification, our findings support the utility of high-dose FNa in the resection of LGG because the imaging correlated to the histology within the regions of lowergrade tumor.

The higher dose of $40 \mathrm{mg} / \mathrm{kg} \mathrm{FNa}$ administered to the patient revealed fine intraoperative imaging characterization using CLE for areas of hypercellularity and tissue. The higher than usual dose of FNa produced unusually excellent contrast for the CLE visualization of the tumor. In conjunction with earlier studies commenting on the potential use of $\mathrm{FNa}$ in the resection of LGG, our report highlights several areas of interest, including the optimal dose of $\mathrm{FNa}$ and which 

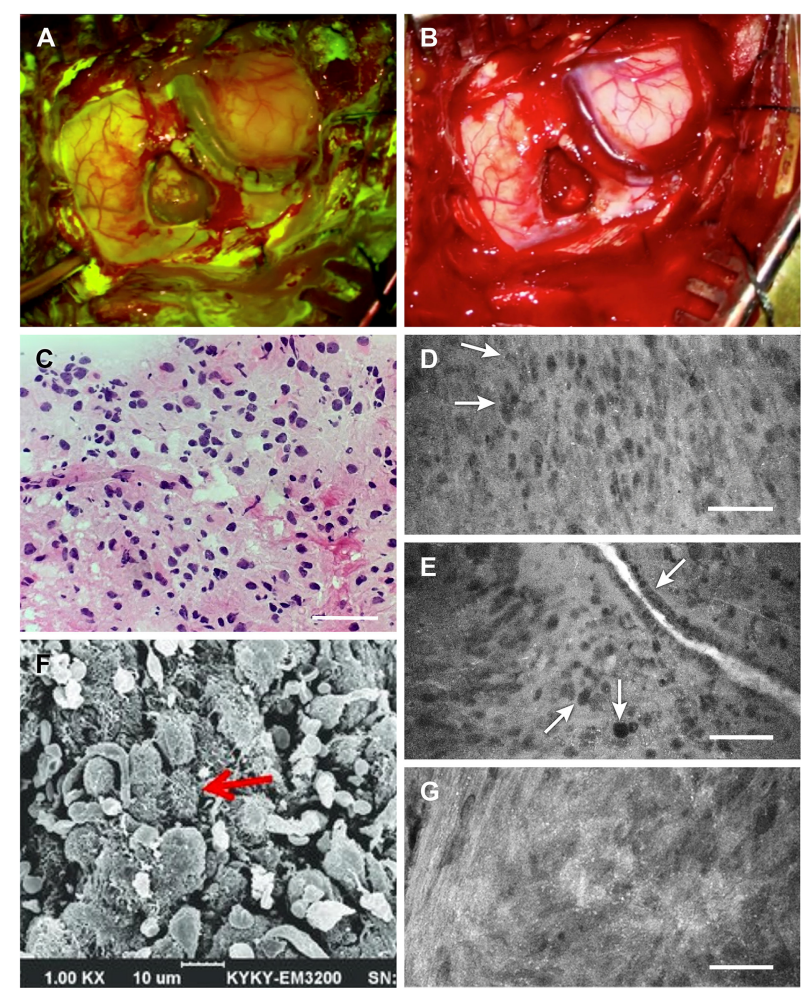

FIGURE 2 | Intraoperative tumor visualization. Exposure during tumor resection with (A) Yellow 560 filter and (B) standard white light. (C) Photograph of intraoperative frozen-tissue section slide interpreted as grade II glioma. (D) Confocal laser endomicroscopy (CLE) image showing subtle discrete cellular aggregates (arrows) within a relatively widespread area of cells interpreted as consistent with lower-grade glioma $1.5 \mathrm{~h}$ after injection of $40 \mathrm{mg} / \mathrm{kg}$ fluorescein sodium (FNa). (E) A vessel 1.5 h after injection of $40 \mathrm{mg} / \mathrm{kg}$ FNa showing irregular endothelium (top arrow), with a focus of cells indicating pleomorphism relative to surrounding cells (center arrow) and what appears to be a mitotic figure (bottom arrow). (F) Scanning electron microscopy (SEM) image showing glioma cell morphology with multiple small vesicles and protrusions. (G) CLE image of another tumor area demonstrates small bright dots that likely represent cell surface vesicles with fluorescein, which corresponds well to the SEM microscopy of the cells. These bright dots were visible throughout the tumor on CLE images. Scale $50 \mu \mathrm{m}$ (D,E,G). Panels (A-E,G) are used with permission from Barrow Neurological Institute, Phoenix, Arizona. Panel (F) is adapted from Lv D, Hu Z, Lu L, Lu H, Xu X. Three-dimensional cell culture: A powerful tool in tumor research and drug discovery. Oncol Lett. 2017;

14(6):6999-7010. Copyright of Institute of Pathology and Southwest Cancer Center and made available under Creative Commons Attribution 3.0 License (https://creativecommons.org/licenses/by/3.0).

grades and characteristics of LGG may be most amenable for FNa-guided resection.

\section{CLE With Low-Dose FNa for Low-Grade Glioma}

Our previous experience with CLE for LGG demonstrated that $\mathrm{FNa}$ contrast was usually inadequate for clear visualization of histologic characteristics, especially $1 \mathrm{~h}$ or longer after administration of FNa (15). In each patient, $5 \mathrm{mg} / \mathrm{kg} \mathrm{FNa}$ was injected, and intraoperative CLE was performed within a few minutes after its administration. In 66 patients, 8 grade II
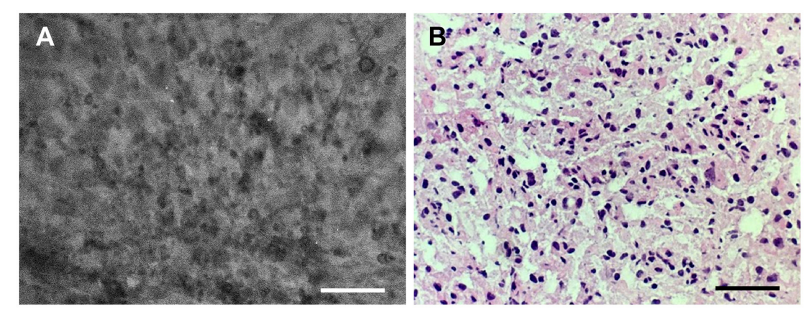

FIGURE 3 | Good correlation of the (A) confocal laser endomicroscopy image collocated with the $\mathbf{( B )}$ hematoxylin-eosin-stained frozen section interpreted as grade II glioma. Scale bars, $50 \mu \mathrm{m}$. Used with permission from Barrow Neurological Institute, Phoenix, Arizona.
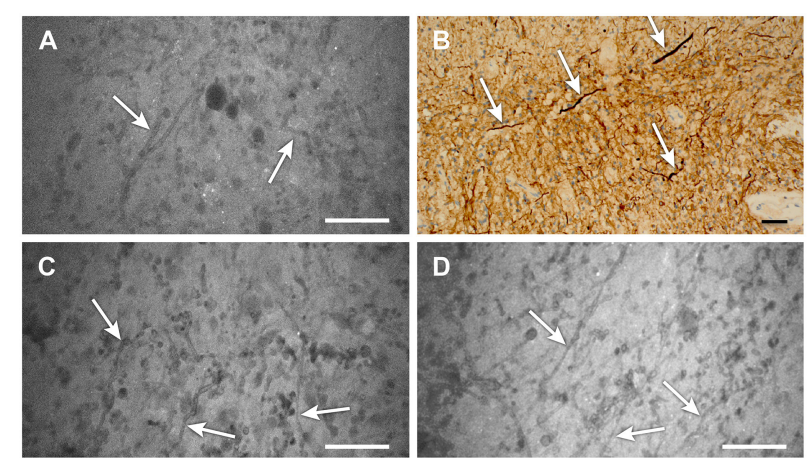

FIGURE 4 | (A-D) Confocal laser endomicroscopy (CLE) images showing areas of low-grade glioma (A,C,D) that reveal distinct strand-like structures or fibers throughout the tumor (scale $50 \mu \mathrm{m}$ ). (B) Image from the same area stained for neurofilaments (arrows) also shows these structures, which are likely swollen axons resulting from tumor edema (scale bar, $20 \mu \mathrm{m})$. We have not previously observed these detailed structures on CLE images with low-dose fluorescein sodium (FNa) staining. Their notable appearance here is likely related to the high dose of FNa used in this case. Used with permission from Barrow Neurological Institute, Phoenix, Arizona.

gliomas were identified, 10 grade III, and 3 grade IV. The exact histopathologic diagnosis of the tumors was not possible for every biopsy or every patient because of the lack of image contrast and clarity to view mitoses adequately enough to count them reliably.

Chen et al. (5) found that, in four gross total resections of LGG, three gliomas were clearly stained after injection of high-dose $\mathrm{FNa}(15-20 \mathrm{mg} / \mathrm{kg})$. In preoperative MRIs, these three tumors also showed enhancement, which is generally found in higher tumor grades. However, in one patient whose LGG was nonenhancing on preoperative MRI, the staining was not clear. Notably, FNa still provided utility in this case because the surgeons could resect around nebulously stained areas, prompting Chen et al. (5) to conclude that FNa may be useful in treating LGG, although this aspect was not specifically evaluated in their investigation.

Schebesch et al. (4) reported five patients with nonenhancing preoperative MRIs but gliomas positive for FET-PET $\left({ }^{18} \mathrm{~F}\right.$ fluoroethyl-L-tyrosine-positron emission tomography), which they referred to as nonenhancing gliomas. All five patients received $5 \mathrm{mg} / \mathrm{kg} \mathrm{FNa}$, and the Yellow 560 filter was used as needed. These investigators found that, despite the lack of 
contrast enhancement on MRI in all patients, all five tumors demonstrated some degree of fluorescence, which correlated with PET metabolic activity. One grade II tumor was visible only under the fluorescence-detecting filter. Final grading identified two LGG and three HGG as grade III. Nonetheless, Schebesch et al. (4) concluded that the incorporation of FNa was helpful both in detecting lesions and in ascertaining their borders, regardless of grade.

Notably, in the report by Schebesch et al. (4), abnormalities were found in the FLAIR and T2 sequences of preoperative MRIs in all five patients in their series. These abnormalities may be sensitive markers of a damaged blood-brain barrier, and they may be present before the tumor becomes contrast-enhancing with progression. In the patient detailed in our case report, preoperative imaging showed similar findings in that the tumor was noncontrast enhancing, but there was a highly abnormal T2/FLAIR signal surrounding the mass. Ultimately, two of the five patients in the Schebesch et al. (4) report were found to have grade III anaplastic oligodendrogliomas, which was the same diagnosis as in our patient. Further studies using FNa may permit elaboration of the characteristics of each tumor subtype under visualization with $\mathrm{FNa}$, and our findings in combination with those previously reported by other authors compel further exploration in future studies.

\section{CLE With High-Dose FNa for Low-Grade Glioma}

Extrapolating from the existing literature and from our observations as detailed in this report, we believe that the application of FNa is influenced by its dosing, both in terms of visualization with or without the Yellow 560 filter and with its beneficial administration in patients with lower grades of glioma. In a study of FNa-guided resection of glioblastoma, Shinoda et al. (20) found that high doses of $\mathrm{FNa}(20 \mathrm{mg} / \mathrm{kg})$ produced usable fluorescence that was viewable under white-light illumination, without the need for an operating microscope Yellow 560 filter. Their report supports the evaluation that higher doses of $\mathrm{FNa}$ can better identify the tumor area and borders. However, their results were obtained from imaging of high-grade lesions with an ostensibly larger degree of blood-brain barrier disruption. Thus, the results may not be translatable to gliomas demonstrating nonenhancement on MRIs. Unlike 5-aminolevulinic acid (5ALA), which does not usually demonstrate cellular uptake in low-grade tumors, high-dose FNa may provide fluorescent visualization and discrimination of the cellular architecture and tumor margin zone.

The question of whether to use $\mathrm{FNa}$ when dealing with either low-grade or high-grade tumors is thus germane for wide-field operative microscopy and CLE imaging. Currently, the intraoperative redosing of $\mathrm{FNa}$ is used as an off-label $\mathrm{FNa}$ application in the United States. In most cases, $\mathrm{FNa}$ is administered at the beginning of the surgery (21), as we have performed it early on. However, exposure of the tumor and the beginning of resection, as well as later inspection of surgical margins, may require many minutes to hours before the use of imaging such as CLE. In our experience, we have begun to delay $\mathrm{FNa}$ administration until after anesthesia induction but immediately before surgical work begins on the tumor, which allows for brighter and clearer images on CLE imaging. However, the timing of $\mathrm{FNa}$ administration is a judgment call because later administration of FNa after accessing the tumor or beginning its surgical resection may highlight surgically damaged tissue as viewed with wide-field imaging, making it almost useless for delineating tumor margin. In most instances, the FNa signal on wide-field imaging is confined to the tumor before it progressively saturates surrounding tissue. This characteristic of FNa can be confusing or disconcerting on wide-field imaging because of the fluorescence of nonrelevant tissues, areas of bleeding, or areas with disruption of tissue during the normal process of surgery.

In many cases where $2-5 \mathrm{mg} / \mathrm{kg} \mathrm{FNa}$ was administered early in the procedure, we acquired CLE imaging that was suboptimal, uninterpretable, or too dark. Thus, we have redosed the FNa to acquire improved CLE images (16). In the current case report, the single $40 \mathrm{mg} / \mathrm{kg} \mathrm{FNa}$ dose produced a signal that remained bright and produced excellent CLE images even $1.5 \mathrm{~h}$ after administration. This case is fortuitous in that it may show implications for very-high-dose FNa administration, where in the past, FNa fluorescence has not been particularly delineative of LGG when used with wide-field operative microscopy.

The two cases where anaphylactic reactions occurred minutes after FNa injection showed decreased blood pressure, bradycardia or tachycardia, and flushing over the infusion anatomical area $(10,11)$. These patients responded to adrenaline, atropine, prednisolone, and dopamine administration. Surgeries were halted, and the patients were moved to the intensive care unit. In both cases, elevated laboratory values of tryptase were found, while in one case, the IgE value was also increased. In both instances, the patients fully recovered, and one report cited the occurrence of such a reaction for the first time in 121 patients injected with FNa for neurosurgery (10).

When the goal is to produce actionable fluorescence image surgical guidance with a wide-field fluorescence detection system, especially when using CLE, a higher dose of FNa may be of more benefit if only one dose is administered at the beginning of an operation. This approach may be particularly useful when using sensitive imaging such as CLE for discriminating the histoarchitecture of tumor margins, especially for LGG tissue that may not be as amenable to 5-ALA fluorescence guidance. Simultaneous use of fluorophores such as 5-ALA and $\mathrm{FNa}$ may be an option for low-grade tumors or nonenhancing tumors. Although 5-ALA has not been shown to delineate lowgrade tumors well, isolated regions of low-grade gliomas often transition to a more metabolically aggressive type. The use of 5-ALA may discriminate those isolated regions, whereas highdose FNa could help define the general tumor area of low-grade tissue and margins. Of course, the logistics of administering the different fluorophores for optimal uptake by tumor cells should be considered. FNa dose-escalation studies in LGG may be useful to define the minimal dosage that allows better interpretation of CLE images to delineate the histologic tumor features and, importantly, the tumor margins or invaded regions. CLE is currently in its infancy regarding in vivo surgical application. 
Cases such as ours may indicate a need for expanded or explorative fluorescence techniques.

\section{CONCLUSIONS}

This case report demonstrates the excellent quality available with dual-fluorescence visualization (wide-field operative microscope Yellow 560 fluorescence navigation and CLE imaging) with a high dose of $\mathrm{FNa}$ in a patient with a nonenhancing glioma up to $1.5 \mathrm{~h}$ after FNa administration. This report supports the investigation of higher doses of $\mathrm{FNa}$ in nonenhancing gliomas and gliomas suspected to be a lower grade that usually do not exhibit 5-ALA-mediated fluorescence. Given the highly informative images, the minimal adverse effects of $\mathrm{FNa}$, and the good postoperative outcome of this case, higher doses of FNa $(20-40 \mathrm{mg} / \mathrm{kg})$ should be considered or trialed with intraoperative CLE.

\section{DATA AVAILABILITY STATEMENT}

The raw data supporting the conclusions of this article will be made available by the authors, without undue reservation.

\section{ETHICS STATEMENT}

The studies involving human participants were reviewed and approved by St. Joseph's Hospital and Medical Center

\section{REFERENCES}

1. Moore GE, Peyton WT, French LA, Walker WW. The clinical use of fluorescein in neurosurgery; the localization of brain tumors. J Neurosurg. (1948) 5:392-8. doi: 10.3171/jns.1948.5.4.0392

2. Acerbi F, Cavallo C, Broggi M, Cordella R, Anghileri E, Eoli M, et al. Fluorescein-guided surgery for malignant gliomas: a review. Neurosurg Rev. (2014) 37:547-57. doi: 10.1007/s10143-014-0546-6

3. Hong J, Chen B, Yao X, Yang Y. Outcome comparisons of high-grade glioma resection with or without fluorescein sodium-guidance. Curr Probl Cancer. (2019) 43:236-44. doi: 10.1016/j.currproblcancer.2018.07.007

4. Schebesch KM, Brawanski A, Doenitz C, Rosengarth K, Proescholdt $\mathrm{M}$, Riemenschneider MJ, et al. Fluorescence-guidance in non-gadolinium enhancing, but FET-PET positive gliomas. Clin Neurol Neurosurg. (2018) 172:177-82. doi: 10.1016/j.clineuro.2018.07.011

5. Chen B, Wang H, Ge P, Zhao J, Li W, Gu H, et al. Gross total resection of glioma with the intraoperative fluorescence-guidance of fluorescein sodium. Int J Med Sci. (2012) 9:708-14. doi: 10.7150/ijms.4843

6. Senders JT, Muskens IS, Schnoor R, Karhade AV, Cote DJ, Smith TR, et al. Agents for fluorescence-guided glioma surgery: a systematic review of preclinical and clinical results. Acta Neurochir (Wien). (2017) 159:15167. doi: 10.1007/s00701-016-3028-5

7. Hara T, Inami M, Hara T. Efficacy and safety of fluorescein angiography with orally administered sodium fluorescein. Am J Ophthalmol. (1998) 126:5604. doi: 10.1016/S0002-9394(98)00112-3

8. Kwan AS, Barry C, McAllister IL, Constable I. Fluorescein angiography and adverse drug reactions revisited: the Lions Eye experience. Clin Exp Ophthalmol. (2006) 34:33-8. doi: 10.1111/j.1442-9071.2006.01136.x

9. Schebesch KM, Proescholdt M, Hohne J, Hohenberger C, Hansen E, Riemenschneider MJ, et al. Sodium fluorescein-guided resection under the YELLOW $560 \mathrm{~nm}$ surgical microscope filter in malignant brain
Institutional Review Board for Human Research. The patients and participants provided written informed consent to participate in this study.

\section{AUTHOR CONTRIBUTIONS}

$\mathrm{EB}$, PN, and MP: study planning and coordination. $\mathrm{EB}$ and IA: acquisition of confocal images. EB, NO, $\mathrm{XZ}$, and IA: processing and organizing of the data and confocal images. EB, XZ, IA, JE, PN, and MP: assessment of confocal images. EB, NO, and MP: writing the draft. MP: final approval. All authors: review of the draft.

\section{FUNDING}

This research was supported by funds from the Barrow Neurological Foundation, the Women's Board of the Barrow Neurological Institute, and the Newsome Family Endowment in Neurosurgery to MP.

\section{ACKNOWLEDGMENTS}

We thank the staff of the Neuroscience Publications office at Barrow Neurological Institute for assistance with manuscript preparation. tumor surgery-a feasibility study. Acta Neurochir (Wien). (2013) 155:6939. doi: 10.1007/s00701-013-1643-y

10. Dilek O, Ihsan A, Tulay H. Anaphylactic reaction after fluorescein sodium administration during intracranial surgery. J Clin Neurosci. (2011) 18:4301. doi: 10.1016/j.jocn.2010.06.012

11. Tanahashi S, Lida H, Dohi S. An anaphylactoid reaction after administration of fluorescein sodium during neurosurgery. Anesth Analg. (2006) 103:503. doi: 10.1213/01.ANE.0000227205.37935.10

12. Belykh E, Shaffer KV, Lin C, Byvaltsev VA, Preul MC, Chen L. Blood-brain barrier, blood-brain tumor barrier, and fluorescence-guided neurosurgical oncology: delivering optical labels to brain tumors. Front Oncol. (2020) 10:739. doi: 10.3389/fonc.2020.00739

13. Schebesch KM, Brawanski A, Hohenberger C, Hohne J. Fluorescein sodium-guided surgery of malignant brain tumors: history, current concepts, and future project. Turk Neurosurg. (2016) 26:185-94. doi: 10.5137/1019-5149.JTN.16952-16.0

14. Belykh E, Miller EJ, Patel AA, Yazdanabadi MI, Martirosyan NL, Yagmurlu $\mathrm{K}$, et al. Diagnostic accuracy of a confocal laser endomicroscope for in vivo differentiation between normal injured and tumor tissue during fluoresceinguided glioma resection: laboratory investigation. World Neurosurg. (2018) 115:e337-e48. doi: 10.1016/j.wneu.2018.04.048

15. Martirosyan NL, Eschbacher JM, Kalani MY, Turner JD, Belykh E, Spetzler RF, et al. Prospective evaluation of the utility of intraoperative confocal laser endomicroscopy in patients with brain neoplasms using fluorescein sodium: experience with 74 cases. Neurosurg Focus. (2016) 40:E11. doi: 10.3171/2016.1.FOCUS15559

16. Belykh E, Zhao X, Ngo B, Farhadi DS, Byvaltsev VA, Eschbacher JM, et al. Intraoperative confocal laser endomicroscopy ex vivo examination of tissue microstructure during fluorescence-guided brain tumor surgery. Front Oncol. (2020) 10:599250. doi: 10.3389/fonc.2020.59 9250 
17. Acerbi F, Pollo B, De Laurentis C, Restelli F, Falco J, Vetrano IG, et al. Ex vivo fluorescein-assisted confocal laser endomicroscopy (CONVIVO(R) System) in patients with glioblastoma: results from a prospective study. Front Oncol. (2020) 10:606574. doi: 10.3389/fonc.2020.606574

18. Hohne J, Schebesch KM, Zoubaa S, Proescholdt M, Riemenschneider MJ, Schmidt NO. Intraoperative imaging of brain tumors with fluorescein: confocal laser endomicroscopy in neurosurgery. Clinical and user experience. Neurosurg Focus. (2021) 50:E19. doi: 10.3171/2020.11.FOCUS20783

19. Olar A, Wani KM, Alfaro-Munoz KD, Heathcock LE, van Thuijl HF, Gilbert $\mathrm{MR}$, et al. IDH mutation status and role of WHO grade and mitotic index in overall survival in grade II-III diffuse gliomas. Acta Neuropathol. (2015) 129:585-96. doi: 10.1007/s00401-015-1398-Z

20. Shinoda J, Yano H, Yoshimura S, Okumura A, Kaku Y, Iwama T, et al. Fluorescence-guided resection of glioblastoma multiforme by using highdose fluorescein sodium. Technical note. J Neurosurg. (2003) 99:597603. doi: $10.3171 /$ jns.2003.99.3.0597
21. Acerbi F, Broggi M, Broggi G, Ferroli P. What is the best timing for fluorescein injection during surgical removal of high-grade gliomas? Acta Neurochir (Wien). (2015) 157:1377-8. doi: 10.1007/s00701-015$2455-\mathrm{z}$

Conflict of Interest: The authors declare that the research was conducted in the absence of any commercial or financial relationships that could be construed as a potential conflict of interest.

Copyright (๑) 2021 Belykh, Onaka, Zhao, Abramov, Eschbacher, Nakaji and Preul. This is an open-access article distributed under the terms of the Creative Commons Attribution License (CC BY). The use, distribution or reproduction in other forums is permitted, provided the original author(s) and the copyright owner(s) are credited and that the original publication in this journal is cited, in accordance with accepted academic practice. No use, distribution or reproduction is permitted which does not comply with these terms. 